\title{
Approximately Self-Consistent Ensemble Density Functional Theory With All Correlations
}

\author{
Tim Gould* \\ Qld Micro- and Nanotechnology Centre, Griffith University, Nathan, Qld 4111, Australia \\ E-mail: t.gould@griffith.edu.au
}

\section{Abstract}

The ability to predict low-lying excited states with the same ease as ground-states would represent a major advance in understanding interactions between light and chemistry, e.g. for solar cells or photocatalysis. Recent theory developments in ensemble density functional theory (EDFT) promise to bring decades of work for ground-states to the practical resolution of excited-state problem - provided newlydiscovered "density-driven correlations" can be dealt with and adequate effective potentials can be found. This Letter introduces simple approximations to both the density-driven correlations and the potential; and shows that EDFT with the $\omega \mathrm{B} 97 \mathrm{X}$ density functional approximation outperforms $\triangle \mathrm{SCF}$ DFT for singlet-triplet gaps in small atoms and molecules. It thus establishes EDFT as a vitally promising tool for low-cost but high-accuracy studies of excited states; and provides a clear route to practical EDFT implementation of arbitrary functional approximations.

\section{Graphical TOC Entry}

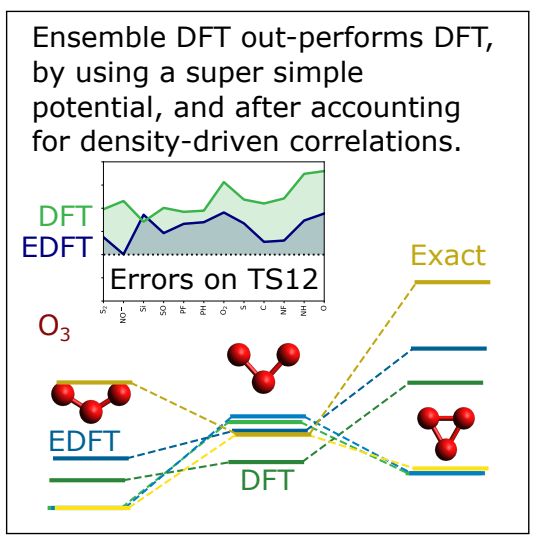


Quantum chemistry has improved our understanding of an enormous range of chemical systems, from biomolecules to battery materials. (Hybrid) density functional theory ${ }^{1+3}$ (DFT) is the primary tool of quantum chemistry, due to its low computational cost and generally good accuracy. Recent developments have seen hybrid DFT calculations reach nearly chemical accuracy, across a wide range of tested systems. $\frac{45}{45}$

Unfortunately, DFT only treats electronic ground-states. This hampers its application to systems involving excited state processes, including those involving interactions with light such as photactivation and charge transfer. Excited state processes are likely to become a vitally important part of future chemistry, due to increasing control over light and the consequent ability to open up new applications. Thus, there is signifcant work on modelling excitation processes (see, e.g. various recent review articles $\left.{ }^{6} \sqrt{8}\right)$. Presently, however, much of this work is fairly high-level, in terms of its numerical cost and/or required expertise.

Time-dependent DFT (TDDFT), and $\triangle \mathrm{SCF}$ DFT are two popular lower-level routes to excited states. In the latter case, ground-state calculations with restricted symmetries are used to find, e.g., the lowest-lying singlet and triplet energies, with the difference being the excitation energy; but this can be quite inaccurate because of "spin contamination" in tripletstates,, 910 and cannot handle excitations of the same symmetry. TDDFT gives a range of excitation energies; but is significantly more costly than DFT, and has some limitations in treating charge transfers ${ }^{11 / 12}$ and double excitations. 13

Ensemble DFT (EDFT) for excited states 14 has recently enjoyed a resurgence as a promising alternative to DFT or TDDFT for treating low-level excited states, including charge transfer and double excitations. $17+33$ In EDFT, the usual DFT approach of seeking a pure-state wavefunction $|\Phi\rangle$ and energy $E_{\Phi}=\langle\Phi|\hat{H}| \Phi\rangle$, is replaced by an equivalent quest for a statistical ensemble $\hat{\Gamma}^{\mathbf{w}}=\sum_{\kappa} w_{\kappa}|\kappa\rangle\langle\kappa|$ and its average energy $\mathcal{E}^{\mathbf{w}}=\sum_{\kappa} w_{\kappa} E_{\kappa}=\operatorname{Tr}\left[\hat{\Gamma}^{\mathbf{w}} \hat{H}\right]$, for orthonormal quantum states $|\kappa\rangle .\left(\left\langle\kappa \mid \kappa^{\prime}\right\rangle=\delta_{\kappa \kappa^{\prime}}\right)$. The set of weights $\mathbf{w}=\left\{w_{\kappa}\right\}$ obey $\sum_{\kappa} w_{\kappa}=1$ and $w_{\kappa} \geq 0$.
EDFT is variational, $14 \mid 15$ just like pure-state DFT. In terms of the versatile Levy constrained minimization ${ }^{34}$ and adiabatic connection ${ }^{35}$ conceptual picture, EDFT simply involves replacing the usual universal functional $F^{\lambda}[n]=$ $\min _{|\Phi\rangle \rightarrow n}\langle\Phi|\hat{T}+\lambda \hat{W}| \Phi\rangle$ by

$$
\mathcal{F}^{\lambda, \mathbf{w}}[n]=\min _{\hat{\Gamma}^{\mathbf{w}} \rightarrow n} \operatorname{Tr}\left[\hat{\Gamma}^{\mathbf{w}}(\hat{T}+\lambda \hat{W})\right],
$$

where $\hat{T}$ is the usual kinetic energy operator and $\hat{W}$ is the electron-electron Coulomb interaction. The average energy $\mathcal{E}^{\mathbf{w}}$ in external potential $v$ can then be found by solving, $\mathcal{E}^{\mathbf{w}}[v]=\min _{n}\left\{\mathcal{F}^{1, \mathbf{w}}[n]+\int d \boldsymbol{r} n(\boldsymbol{r}) v(\boldsymbol{r})\right\}$, where $n(\boldsymbol{r})=\operatorname{Tr}\left[\hat{\Gamma}^{\mathbf{w}} \hat{n}\right]=\sum_{\kappa} w_{\kappa} n_{\kappa}(\boldsymbol{r})$ is the average density of the ensemble. Variational principles give $E_{\kappa} \leq E_{\kappa^{\prime}}$ for $w_{\kappa}>w_{\kappa^{\prime}}$, i.e. the energies become ordered. Excitation energies can be found by varying weights, and taking differences or derivatives. Note, calligraphic letters indicate ensemble density functionals and explicit dependence on $\mathbf{w}$ is henceforth dropped for notational simplicity.

Recent work ${ }^{24 \mid 29}$ showed how Eq. (1) can be used to provide EDFT analogs for the usual DFT functionals. EDFT has a kinetic energy,

$$
\mathcal{T}_{s}[n]=\mathcal{F}^{0}[n] \equiv \sum_{\kappa} w_{\kappa} T_{s, \kappa}
$$

and Hartree-exchange (Hx) functional,

$$
\mathcal{E}_{\mathrm{Hx}}[n]=\lim _{\lambda \rightarrow 0^{+}} \frac{F^{\lambda}[n]-\mathcal{T}_{s}[n]}{\lambda} \equiv \sum_{\kappa} w_{\kappa} \Lambda_{\mathrm{Hx}, \kappa},
$$

like usual. Except: i) $T_{s, \kappa}=\left\langle\kappa_{s}|\hat{T}| \kappa_{s}\right\rangle$ and $\Lambda_{\mathrm{Hx}, \kappa}=\left\langle\kappa_{s}|\hat{W}| \kappa_{s}\right\rangle$ differ for each member $\left|\kappa_{s}\right\rangle$ of the KS ensemble; and ii) $\Lambda_{\mathrm{Hx}, \kappa}$ can inherit multi-configuration qualities to account for different spin symmetries. 24

It also has a correlation energy term $\mathcal{E}_{\mathrm{c}}=$ $\mathcal{F}^{1}-\mathcal{T}_{s}-\mathcal{E}_{\mathrm{Hx}}$. However, unlike pure state DFT,

$$
\mathcal{E}_{\mathrm{c}}[n]=\mathcal{E}_{\mathrm{c}}^{\mathrm{SD}}[n]+\mathcal{E}_{\mathrm{c}}^{\mathrm{DD}}[n],
$$

involves two terms: $\mathcal{E}_{\mathrm{c}}^{\mathrm{SD}}[n]=\sum_{\kappa} w_{\kappa} E_{\mathrm{c}, \kappa}^{\mathrm{SD}}$ and $\mathcal{E}_{\mathrm{c}}^{\mathrm{DD}}[n]=\sum_{\kappa} w_{\kappa} E_{\mathrm{c}, \kappa}^{\mathrm{DD}}$. The state-driven (SD) correlation energy terms $E_{\mathrm{c}, \kappa}^{\mathrm{SD}}$ are like the usual 
DFT correlation energy, and are invariant to $\mathbf{w}$ in certain cases, including those studied here. The density-driven (DD) terms $E_{\mathrm{c}, \kappa}^{\mathrm{DD}}$ are unique to $\operatorname{EDFT}$ (i.e., $\mathcal{E}_{\mathrm{c}}^{\mathrm{DD}}=0$ in pure-states) and carry a non-trivial dependence on $\mathbf{w}$ due to the inability of the KS ensemble members to simultaneously reproduce the densities and orbitals of all interacting ensemble members. $\frac{29}{29}$

Putting everything together gives,

$\mathcal{E}[v]=\min _{n}\left\{\mathcal{T}_{s}[n]+\mathcal{E}_{\mathrm{Hxc}}[n]+\int d \boldsymbol{r} n(\boldsymbol{r}) v(\boldsymbol{r})\right\}$,

where $\mathcal{E}_{\mathrm{Hxc}}=\mathcal{E}_{\mathrm{Hxc}}^{\mathrm{SD}}+\mathcal{E}_{\mathrm{c}}^{\mathrm{DD}}$ and

$$
\mathcal{E}_{\mathrm{Hxc}}^{\mathrm{SD}}=\sum_{\kappa} w_{\kappa}\left[\Lambda_{\mathrm{Hx}, \kappa}+E_{\mathrm{c}, \kappa}^{\mathrm{SD}}\right] \equiv \sum_{\kappa} w_{\kappa} E_{\mathrm{Hxc}, \kappa}^{\mathrm{SD}},
$$

Both $\Lambda_{\mathrm{Hx}, \kappa}$ and $E_{\mathrm{c}, \kappa}$ are pure-state-like. Thus, a useful strategy is to adapt existing density functional approximations (DFAs) for use in $E_{\mathrm{Hxc}, \kappa}^{\mathrm{SD}}$. The key outstanding challenges are then how to modify existing approximations to deal with different ensemble members, and how to evaluate $\mathcal{E}_{\mathrm{c}}^{\mathrm{DD}}$. Both will be tackled later.

Once approximated, the energy functional, (5), must then be minimized on the density. Minimization of (5) involves finding a set of KS orbitals $\phi_{i}$ that obey the usual spinindependent $\mathrm{KS}$ equation,

$$
\left[-\frac{1}{2} \nabla^{2}+v_{s}[n](\boldsymbol{r})\right] \phi_{i}(\boldsymbol{r})=\epsilon_{i} \phi_{i}(\boldsymbol{r}),
$$

where $v_{s}=v+\frac{\delta \mathcal{E}_{\mathrm{Hxc}}}{\delta n}$. Then, $n(\boldsymbol{r})=\sum_{i} f_{i} \phi_{i}(\boldsymbol{r})^{2}$, where $0 \leq f_{i} \leq 2$ is the average occupation factor of orbital $\phi_{i}$, which obey $\sum_{i} f_{i}=N$ for $N$ electrons, and which depend on $\mathbf{w} . \underline{24 / 36}$ Unfortunately, the EDFT density functional $\mathcal{E}_{\mathrm{Hxc}}$ has non-trivial dependencies on orbitals, whether treated exactly or approximately. Thus, unlike conventional DFT, direct density functional derivatives $\delta \mathcal{E}_{\mathrm{Hxc}} / \delta n$ cannot easily be found.

To circumvent this problem, one can use a generalized KS (GKS) formalism $\frac{37}{37}$ or an optimized effective potential ${ }^{[38] 39}$ (OEP). Both approaches have challenges. This work adopts a different approach - any GKS treatment re- quired by the DFA is done as per the groundstate case. An additional potential is then introduced, which is approximated by a super simple OEP (SSOEP), introduced later. Consequently, Eq. (7) can be solved using existing machinery implemented in any molecular code.

The rest of this Letter proceeds as follows. First, a methodology is introduced to adapt the successful $\omega$ B97X DFA ${ }^{40}$ to EDFT. This necessitates dealing with the DD correlation energy and SSOEP, which are discussed. Next, the method is assessed on several tests to illustrate how EDFT usually outperforms DFT for excitations, even when treated at the same level of theory. Finally, some conclusions are drawn.

Theory: Application of EDFT relies on approximating $\mathcal{E}_{\mathrm{Hxc}}=\mathcal{E}_{\mathrm{Hx}}+\mathcal{E}_{\mathrm{c}}^{\mathrm{SD}}$, like DFT. Ideally, one would use existing approximations, which have already been optimized for ground-states. This work extends the successful $\omega$ B97X functional of Chai and Head-Gordon $\underline{40}$ to EDFT. Thus, one obtains,

$$
\mathcal{E}^{\mathrm{SD}}=\mathcal{T}_{s}+\sum_{\kappa} w_{\kappa} E_{\mathrm{Hxc}, \kappa}^{\omega \mathrm{B} 97 \mathrm{X}}+\int n v d \boldsymbol{r}
$$

for the SD energy, with Hxc terms,

$$
E_{\mathrm{Hxc}, \kappa}^{\omega \mathrm{B} 97 \mathrm{X}}=\Lambda_{\mathrm{Hx}, \kappa}-0.8423 \Lambda_{\mathrm{x}, \kappa}^{\mathrm{sr}}+E_{\mathrm{xc}, \kappa}^{\mathrm{DFA}} .
$$

The use of $\Lambda_{\mathrm{Hx}, \kappa}$ indicates that spin symmetries are properly accounted for. The above and what follows can, of course, be adapted to any DFA of (hybrid) generalized-gradient approximation (GGA) character.

Any (range-separated) hybrid GGA requires three key inputs: densities $n$, spin-polarizations $\zeta$, and (range-separated) Hartree and exchange energies $\Lambda_{\mathrm{Hx}}$. Each KS state $\left|\kappa_{s}\right\rangle$ is trivially associated with a density $n_{s, \kappa}=\left\langle\kappa_{s}|\hat{n}| \kappa_{s}\right\rangle$. However, $\zeta=0$ in a spin-restricted formalism. Instead, $\zeta_{s, \kappa}=\left(1-2 P_{2, \kappa} / n_{s, \kappa}^{2}\right)^{\frac{1}{2}}$ is employed, per previous work, ${ }^{29141}$ where $P_{2, \kappa}$ is the KS on-top pair-density. Together, these terms are used to semi-locally approximate $E_{\mathrm{xc}, \kappa}^{\mathrm{DFA}} \equiv E_{\mathrm{xc}}\left[n_{s, \kappa}, \zeta_{s, \kappa}\right]$ for short-range exchange and all correlations.

$\Lambda_{\mathrm{Hx}, \kappa}$ is an orbital functional defined in eq. (3). The range-separated Hartree-exchange energy $\Lambda_{\mathrm{x}, \kappa}^{\mathrm{sr}}$ is found by adopting the same 
functional form, but replacing the Coulomb potential $1 / r$ by its short-range counterpart $\operatorname{erfc}(\omega r) / r$ (with $\omega=0.3$ a.u. $\left.^{-1}\right)$. Then, $\Lambda_{\mathrm{x}, \kappa}^{\mathrm{sr}}=$ $\Lambda_{\mathrm{Hx}, \kappa}^{\mathrm{sr}}-E_{\mathrm{H}}^{\mathrm{sr}}\left[n_{s, \kappa}\right]$ where $E_{\mathrm{H}}$ is the usual Hartree energy expression. Full details are in the Supporting Information.

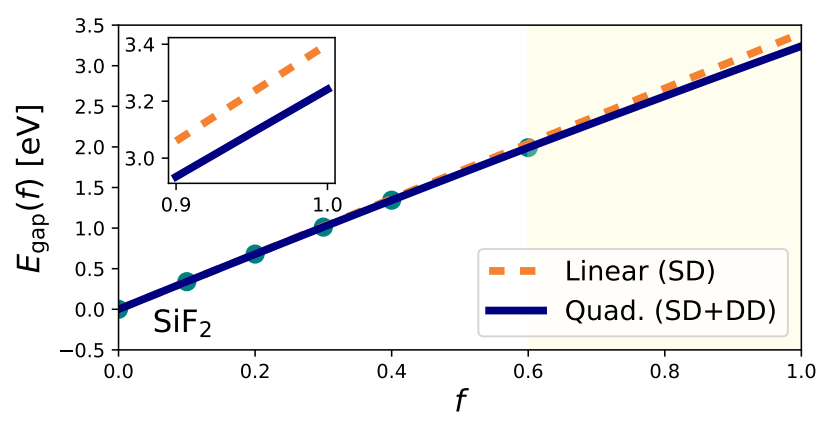

Figure 1: Illustration of the exrapolation procedure applied to $\mathrm{SiF}_{2}$. The inset zooms into the DD contribution to the singlet-triplet gap. Light shading indicates the extrapolation region $W \geq 0.6$.

This establishes the SD energy approximation. However, DD correlations must also be accounted for. Previous work introduced a direct approximation for DD terms. ${ }^{29}$ Here, $\mathcal{E}_{\mathrm{c}}^{\mathrm{DD}}$ is found by a parameter-free extrapolation, which is conceptually similar to a technique recently used to calculate DD correlations for $S-P$ and $S-S$ transitions in atoms. 30 A full derivation of the approach is rather long, so is left to the Supporting Information. Key steps are as follows: 1) Recognise that $\mathcal{E}^{w}=\mathcal{E}^{\mathrm{SD}, w}+\mathcal{E}_{\mathrm{c}}^{\mathrm{DD}, w}$ is linear in $w$ so that the excitation energy $\Delta E=E_{\mathrm{ts}}-E_{\mathrm{ss}}=\partial_{w} \mathcal{E}^{w}, \forall w-$ where $\mathcal{E}^{w} \equiv$ $\mathcal{E}^{\{1-w, w\}}, \mathcal{E}^{\mathrm{SD}, w} \equiv \mathcal{E}^{\omega \mathrm{B} 97 \mathrm{X}, w}$ is evaluated using eq. (8), and $\mathcal{E}_{\mathrm{c}}^{\mathrm{DD}, w}$ is unknown; 2) Approximate the $\mathrm{SD}$ excitation energy as $\Delta E^{\mathrm{SD}}=$ $E_{\mathrm{ts}}^{\mathrm{SD}}-E_{\mathrm{sS}}^{\mathrm{SD}}=\left.\partial_{w} \mathcal{E}^{\omega \mathrm{B} 97 \mathrm{X}, w}\right|_{w=0}$ so that it corresponds to a "typical" EDFT calculation using orbitals of the singlet ground-state; 3) Recognise that $\mathcal{E}^{0}=\mathcal{E}^{\omega \mathrm{B} 97 \mathrm{X}, 0}$ and $\mathcal{E}^{1}=\mathcal{E}^{\omega \mathrm{B} 97 \mathrm{X}, 1}$ in the pure-state limits.

This gives singlet-triplet gaps

$$
\begin{aligned}
\Delta E & =\operatorname{ext}_{w \rightarrow 1} \mathcal{E}^{\omega \mathrm{B} 97 \mathrm{X}, w}-\underset{w \rightarrow 0}{\operatorname{ext}} \mathcal{E}^{\omega \mathrm{B} 97 \mathrm{X}, w}, \\
\Delta E^{\mathrm{SD}} & =\left.\partial_{w} \mathcal{E}^{\omega \mathrm{B} 97 \mathrm{X}, w}\right|_{w=0},
\end{aligned}
$$

so that the total DD contribution to the excita- tion is $\Delta \mathcal{E}_{\mathrm{c}}^{\mathrm{DD}}=\Delta E-\Delta E^{\mathrm{SD}}$. Here, ext $_{w \rightarrow 1}$ involves fitting a quadratic (chosen to avoid over-fitting) to values of $\mathcal{E}^{\omega \mathrm{B} 97 \mathrm{X}, w_{i}}$ found for $w_{i} \in[0,0.6]$, then extrapolating to $w=1$ (for numerical consistency, the same fit is also used to calculate the singlet state at $w=0$ ). This division into SD/DD differs very slightly from the formal definition, 29 but may be more suited to studying DFAs. The extrapolation is illustrated in Figure 1 .

The final approximation is the super simple OEP (SSOEP), to account for the effective potential. This involves two steps: 1) try a variety of conventional DFAs (here, PBE, $\underline{42}$ $\mathrm{PBE}^{43}$ and $\omega \mathrm{B} 97 \mathrm{X}$ - in this last case, the effective Hamiltonian also includes GKS terms) to obtain a potential approximation $\tilde{v}_{s}^{0}$ (e.g., $\tilde{v}_{s}^{0}=\tilde{v}_{s}^{\mathrm{PBE} 0}$ ) whose orbitals $\phi_{i}^{0}[$ using (7)] and densitiy $n^{0}$ give the lowest energy [using (8)]; 2) Find $\xi$ such that the orbitals $\phi_{i}^{\xi}$ and density $n^{\xi}$ [using (7)] from the potential

$$
\tilde{v}_{s}^{\xi}(\boldsymbol{r})=\tilde{v}_{s}^{0}(\boldsymbol{r})+\xi \frac{n_{\mathrm{gs}^{\prime}}^{0}(\boldsymbol{r})}{n^{0}(\boldsymbol{r})}
$$

minimize the energy $\mathcal{E}^{\xi}$ [using (8)]. Here, $n_{\mathrm{gs}^{\prime}}^{0}(\boldsymbol{r})=\sum_{i \leq N / 2} f_{i} \phi_{i}^{0}(\boldsymbol{r})^{2}$ is the ground-statelike density, with excited state orbitals excluded. This second step involves an ansatz for the potential inspired by a previous finding for fractional EDFT that the exact KS potential is approximately given by a similar expression see eq. 15, of Gould and Toulouse.44

This approach avoids spin contamination when treating singlet or triplet states - unlike $\Delta \mathrm{SCF}$ DFT, because $\phi_{i \uparrow}(\boldsymbol{r})=\phi_{i \downarrow}(\boldsymbol{r})$ for all orbitals. It thus follows that DFT and EDFT results for singlet ground-states are the same; but triplet- states differ. Implementation requires only small modification of existing code, and iterates to a solution in around five steps.

Together, our approximations for the DD correlation energy and effective potential provide an overarching methodology that lets a general DFA (here $\omega$ B97X) be applied to EDFT problems with the same scaling as conventional DFT, albeit with a larger pre-factor from the density-driven correlations $(\times 6$, here $)$ and SSOEP $(\times 8$, here). It is much cheaper than 


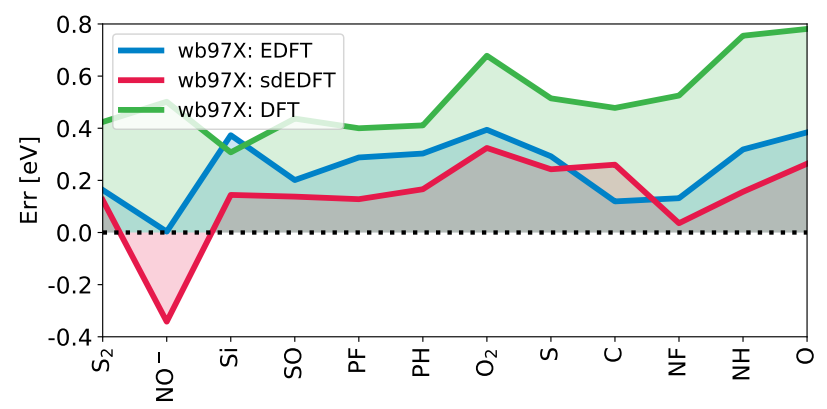

Figure 2: Errors $[\mathrm{eV}]$ in the triplet-singlet gap for the TS12 benchmark set in DFT (green), EDFT (blue) and sdEDFT (red).

the additional $O(N)$ overhead of TDDFT calculations. The approach is also "black box", making it easy to use without specifying active states or intuiting the processes involved.

Results: With the theory established, the approach is validated by applying it to various tests on small molecules. Tests are restricted to singlet-triplet gaps, as these allow direct comparison with $\triangle \mathrm{SCF}$ DFT at the same level of theory.

The TS12 benchmark set of small tripletsinglet gaps in small molecules forms the first test. ${ }^{[4]}$ The small gaps in these systems make them particularly problematic for DFT, as symmetry breaking can lead to substantial errors. [Note, the triplet-state is the ground-state in these systems. This does not change the formalism.]

Figure 2 shows errors from DFT (using $\triangle \mathrm{SCF}), \mathrm{EDFT}$ and state-driven EDFT calculations without DD correlations (sdEDFT). Overall, EDFT, with a mean absolute error (MAE) of $0.25 \mathrm{eV}$ offers a two-fold improvement over DFT, with MAE $0.52 \mathrm{eV}$. EDFT without DD correlations slightly out-performs the full EDFT treatment, with MAE $0.19 \mathrm{eV}$.

It is clear from these results that EDFT can appropriately deal with excitations, as one would expect from its consistent treatment of ground- and excited-states. What is notable is that including the DD correlation energy does not always improve things, despite its formal justification. However, this is easy to explain. In these molecules the DD correlation energy is typically small $(<0.2 \mathrm{eV})$, which is smaller than the underlying error of the DFA. Thus, any small positive/negative DD correction to an over/underprediction by the DFAs will make things slightly worse. Importantly, in the one case $\left(\mathrm{NO}^{-}\right)$where the $\mathrm{DD}$ term makes a big difference to the energy, it improves things.

To supplement TS12, vertical and adiabatic singlet-triplet gaps are calculated for three small molecules $\left(\mathrm{SO}_{2}, \mathrm{SiF}_{2}, \mathrm{CHCl}\right.$ and $\mathrm{CSHH}$ ) from the Loos-Jacquemin benchmark $\operatorname{set}^{45}$ (LJ19). [Note, there is a mistake in the $\mathrm{SO}_{2}$ triplet structure reported by Loos and Jacquemin. This work uses a structure provided by the authors.]

Results for vertical (using the ground-state geometry) and adiabatic (using the optimal geometries for ground- and excited states) excitations are reported in Table 1. On average, DFT slightly (by $0.06 \mathrm{eV}$ on MAE) outperforms EDFT for vertical excitations. But, DFT does significantly better for adiabatic excitations (by $0.22 \mathrm{eV}$ on MAE). The poor performance of EDFT for adiabatic excitations is mostly due to a very poor prediction for $\mathrm{CSHH}$, which is out by around $40 \%$. sdEDFT gives the worst results in all cases, meaning that, for these molecules, density-driven correlations are both quantitatively important and systematically beneficial.

Finally, a particularly challenging test is applied - predicting key excitations of stronglycorrelated ozone. This involves computing singlet-triplet gaps at three selected geometries: open minimum (OM), transition state (TS) and ring minimum (RM). These represent key steps in the ozone excitation process that were recently benchmarked using high-level theory. $₫ 7$ This problem represents the sort of application for which EDFT will likely prove most useful, e.g. to simultaneously predict multiple states for use in beyond-Born-Oppenheimer dynamics. The TS geometry is a very stringent test, due to the degeneracy of singlet- and triplet- states.

Figure 3 shows that EDFT clearly outperforms regular DFT and gives semi-quantitive agreement with the exact solution. EDFT almost predicts the degeneracy between the singlet- (ss) and triplet-states (ts) in TS, unlike DFT. By contrast, $\triangle \mathrm{SCF}$ DFT does a poor job 
Table 1: Vertical and adiabatic excitation energies $[\mathrm{eV}]$ from DFT, EDFT and sdEDFT (sdE). Benchmarks from LJ19. $₫ 5$

\begin{tabular}{|c|c|c|c|c|c|c|c|c|c|c|c|c|c|c|}
\hline & \multicolumn{7}{|c|}{ Vertical } & \multicolumn{7}{|c|}{ Adiabatic } \\
\hline & Bench & DFT & Err & EDFT & Err & $\mathrm{sdE}$ & Err & Bench & DFT & Err & EDFT & Err & $\mathrm{sdE}$ & Err \\
\hline $\mathrm{SiF}_{2}$ & 3.30 & 3.76 & 0.46 & 3.50 & 0.20 & 3.76 & 0.46 & 3.20 & 3.26 & 0.06 & 3.38 & 0.18 & 3.63 & 0.43 \\
\hline $\mathrm{SO}_{2}$ & 3.45 & 3.37 & -0.08 & 3.51 & 0.06 & 3.62 & 0.17 & 3.03 & 2.88 & -0.15 & 3.22 & 0.19 & 3.32 & 0.29 \\
\hline $\mathrm{CSHH}$ & 1.96 & 1.70 & -0.26 & 2.47 & 0.51 & 2.58 & 0.62 & 1.86 & 1.62 & -0.24 & 2.58 & 0.73 & 2.68 & 0.82 \\
\hline $\mathrm{CHCl}$ & 0.58 & 0.46 & -0.12 & 0.98 & 0.40 & 1.14 & 0.56 & 0.24 & 0.11 & -0.13 & 0.59 & 0.35 & 0.73 & 0.49 \\
\hline MAE & & & 0.23 & & 0.29 & & 0.45 & & & 0.14 & & 0.36 & & 0.51 \\
\hline
\end{tabular}

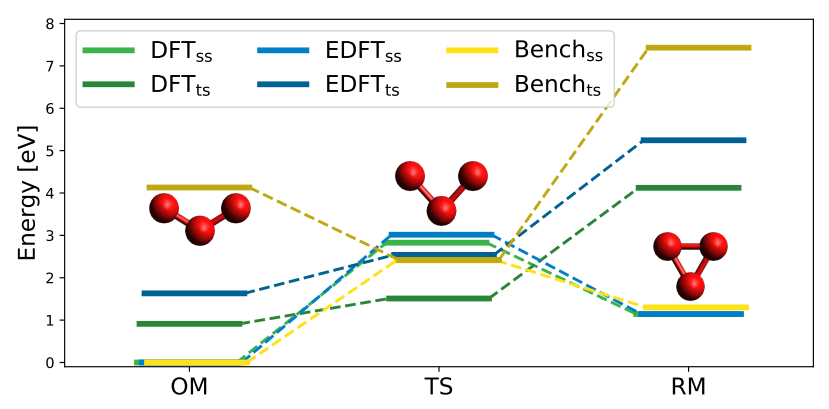

Figure 3: Energy levels for three geometries of ozone in DFT (green) and EDFT (blue) and benchmark ${ }^{47}$ (yellow) calculations. Lighter colours indicate the ground-state (singlet) and darker colours indicate the excited state (triplet). Geometries are also shown.

of the triplet state in all cases. EDFT, unlike DFT, is thus useable for estimating dynamics. All relevant energies are shown in the Supporting Information. Note, in nearly-degenerate TS there is also a small $(0.2 \mathrm{eV})$ difference between singlet states calculated using DFT and EDFT, because the latter are obtained using a quadratic fit on $w$.

sdEDFT (not shown) gives very similar results to full EDFT for OM and RM geometries. But for TS it predicts a significant gap of $5.1 \mathrm{eV}$, compared to a zero gap in the benchmark, and gaps of $0.13 \mathrm{eV}$ for full EDFT and $1.3 \mathrm{eV}$ for DFT. Thus, inclusion of the DD correlation energy term is vital.

Conclusions: In summary, this letter reports approximately self-consistent and fully correlated ensemble DFT (EDFT) calculations using the $\omega \mathrm{B} 97 \mathrm{X} \mathrm{DFA}$, and including recentlydiscovered density-driven (DD) correlation energy terms. $\frac{29}{29}$ Obtaining these results required the introduction of two methodological innova- tions: 1) a way to deal with DD correlations [eq. (10)]; 2) a way to deal with self-consistency [eq. (12)].

Results showed that EDFT improved on $\triangle \mathrm{SCF}$ DFT for the computation of low-lying excited states in almost all cases, and is thus a promising alternative for low-cost calculations. For the TS12 benchmark set, EDFT gave a two-fold improvement over DFT using the same $\omega$ B97X approximation. Small atom excitations (vertical and adiabatic) from LJ19 were reproduced reasonable well by both DFT and EDFT, except the latter failed badly for CSHH. Most promisingly, EDFT (but not sdEDFT) significantly out-performed DFT for difficult ozone excitations.

Including DD correlations in our ensemblised $\omega \mathrm{B} 97 \mathrm{X}$ calculations is vital in some systems, (e.g., $\mathrm{NO}^{-}$, ozone TS) but slightly detrimental in others (e.g. Si, PF). Since these are small energies, and $\omega \mathrm{B} 97 \mathrm{X}$ is an "uncontrolled" (nonvariational) approximation, some variation is to be expected. Results are certainly consistent with chemical intuition. A deeper conceptual understanding of DD terms (e.g., understanding when ground-state orbitals are poor for excited-states) might point the way to improved treatment of excitations generally, not just in EDFT - analogous to how a better understanding of dispersion corrections has improved treatment of weak binding generally. This problem should be investigated.

I speculate that the poor showing for EDFT in CSHH might reflect deficiencies in the super simple OEP. Better, low-cost solutions might be required for general cases. Theoretical and numerical work along these lines is being pursued.

Finally, unlike $\triangle \mathrm{SCF}$ DFT, the EDFT 
scheme proposed here can handle singletsinglet excitations as readily as singlet-triplet excitations. .30 Their quality will be investigated in future work, which must accommodate recent developments in Hartree theory for ensembles. 48

\section{Computational Methods}

All calculations were performed in a custom Python3 code, based on Psi4/NumPy. ${ }^{49[50}$ The code used standard routines therefrom wherever possible. Both DFT and EDFT calculations of the TS12 benchmark set were performed with the aug-cc-pVQZ basis set. 151 These calculations were repeated with aug-ccpVTZ, which was found to give similar results (within $0.04 \mathrm{eV}$ of aug-cc-pVQZ, except for $\mathrm{NO}^{-}$). Since no other anions were tested, augcc-pVTZ was thus used for the remaining calculations. Spatial symmetries were allowed to break, to avoid the additional ensemble effects required to preserve them.

EDFT calculations used ensemble weights $w \in\{0.0,0.1,0.2,0.3,0.4,0.6\}$ in the quadratic extrapolation to $w=1$. sdEDFT calculations used a finite difference $\left.\partial_{w} E\right|_{w=0} \approx\left(E^{w=0.1}-\right.$ $\left.E^{w=0.0}\right) / 0.1$ to approximate the gradient at $w=$ 0 .

Acknowledgement This work was supported by the Australian Research Council (DP200100033); the National Computational Merit Allocation Scheme (NCMAS project ca11) for National Computational Infrastructure, Australia; and the Queensland Cyber Infrastructure Foundation.

\section{Supporting Information Avail- able}

Includes: 1) Full working for the implementation of the $\omega \mathrm{B} 97 \mathrm{X}$ density functional approximation for ensembles. 2) Details of the densitydriven correlation calculation. 3) Tabulated data for calculations.

\section{References}

(1) Hohenberg, P.; Kohn, W. Inhomogeneous Electron Gas. Phys. Rev. 1964, 136, B864-B871.

(2) Kohn, W.; Sham, L. J. Self-Consistent Equations Including Exchange and Correlation Effects. Phys. Rev. 1965, 140, A1133-A1138.

(3) Kim, K.; Jordan, K. D. Comparison of Density Functional and MP2 Calculations on the Water Monomer and Dimer. J. Phys. Chem. 1994, 98, 10089-10094.

(4) Goerigk, L.; Hansen, A.; Bauer, C.; Ehrlich, S.; Najibi, A.; Grimme, S. A look at the density functional theory zoo with the advanced GMTKN55 database for general main group thermochemistry, kinetics and noncovalent interactions. Phys. Chem. Chem. Phys. 2017, 19, 3218432215 .

(5) Mardirossian, N.; Head-Gordon, M. Thirty years of density functional theory in computational chemistry: an overview and extensive assessment of 200 density functionals. Mol. Phys. 2017, 0, 1-58.

(6) Crespo-Otero, R.; Barbatti, M. Recent Advances and Perspectives on Nonadiabatic Mixed Quantum-Classical Dynamics. Chem. Rev. 2018, 118, 7026-7068.

(7) Ghosh, S.; Verma, P.; Cramer, C. J.; Gagliardi, L.; Truhlar, D. G. Combining Wave Function Methods with Density Functional Theory for Excited States. Chem. Rev. 2018,

(8) Lischka, H.; Nachtigallová, D.; Aquino, A. J. A.; Szalay, P. G.; Plasser, F.; Machado, F. B. C.; Barbatti, M. Multireference Approaches for Excited States of Molecules. Chem. Rev. 2018, 118, 72937361 .

(9) Baker, J.; Scheiner, A.; Andzelm, J. Spin contamination in density functional the- 
ory. Chem. Phys. Lett. 1993, 216, 380388 .

(10) Wittbrodt, J. M.; Schlegel, H. B. Some reasons not to use spin projected density functional theory. J. Chem. Phys. 1996, 105, 6574-6577.

(11) Maitra, N. T. Undoing static correlation: Long-range charge transfer in timedependent density-functional theory. $J$. Chem. Phys. 2005, 122, 234104.

(12) Maitra, N. T. Charge transfer in timedependent density functional theory. $J$. Phys.: Cond. Matter 2017, 29, 423001.

(13) Elliott, P.; Goldson, S.; Canahui, C.; Maitra, N. T. Perspectives on doubleexcitations in TDDFT. Chem. Phys. 2011, 391, 110-119.

(14) Gross, E. K. U.; Oliveira, L. N.; Kohn, W. Rayleigh-Ritz variational principle for ensembles of fractionally occupied states. Phys. Rev. A 1988, 37, 2805-2808.

(15) Gross, E. K. U.; Oliveira, L. N.; Kohn, W. Density-functional theory for ensembles of fractionally occupied states. I. Basic formalism. Phys. Rev. A 1988, 37, 28092820 .

(16) Oliveira, L. N.; Gross, E. K. U.; Kohn, W. Density-functional theory for ensembles of fractionally occupied states. II. Application to the He atom. Phys. Rev. A 1988, 37, 2821-2833.

(17) Filatov, M.; Shaik, S. A spin-restricted ensemble-referenced Kohn-Sham method and its application to diradicaloid situations. Chem. Phys. Lett. 1999, 304, 429437.

(18) Pribram-Jones, A.; Yang, Z.-h.; Trail, J. R.; Burke, K.; Needs, R. J.; Ullrich, C. A. Excitations and benchmark ensemble density functional theory for two electrons. J. Chem. Phys. 2014, 140.
(19) Yang, Z.-h.; Trail, J. R.; PribramJones, A.; Burke, K.; Needs, R. J.; Ullrich, C. A. Exact and approximate KohnSham potentials in ensemble densityfunctional theory. Phys. Rev. A 2014, 90, 042501.

(20) Filatov, M.; Huix-Rotllant, M.; Burghardt, I. Ensemble density functional theory method correctly describes bond dissociation, excited state electron transfer, and double excitations. J. Chem. Phys. 2015, 142, 184104.

(21) Senjean, B.; Knecht, S.; Jensen, H. J. A.; Fromager, E. Linear interpolation method in ensemble Kohn-Sham and rangeseparated density-functional approximations for excited states. Phys. Rev. A 2015, 92, 012518.

(22) Filatov, M. In Density-Functional Methods for Excited States; Ferré, N., Filatov, M., Huix-Rotllant, M., Eds.; Springer International Publishing: Cham, 2016; pp 97-124.

(23) Yang, Z.-h.; Pribram-Jones, A.; Burke, K.; Ullrich, C. A. Direct Extraction of Excitation Energies from Ensemble DensityFunctional Theory. Phys. Rev. Lett. 2017, 119, 033003.

(24) Gould, T.; Pittalis, S. Hartree and Exchange in Ensemble Density Functional Theory: Avoiding the Nonuniqueness Disaster. Phys. Rev. Lett. 2017, 119, 243001.

(25) Senjean, B.; Fromager, E. Unified formulation of fundamental and optical gap problems in density-functional theory for ensembles. Phys. Rev. A 2018, 98, 022513 .

(26) Sagredo, F.; Burke, K. Accurate double excitations from ensemble density functional calculations. J. Chem. Phys. 2018, $149,134103$.

(27) Filatov, M.; Min, S. K.; Kim, K. S. Nonadiabatic dynamics of ring opening in 
cyclohexa-1, 3-diene described by an ensemble density-functional theory method. Mol. Phys. 2018, 1-14.

(28) Gould, T.; Kronik, L.; Pittalis, S. Charge transfer excitations from exact and approximate ensemble Kohn-Sham theory. $J$. Chem. Phys. 2018, 148, 174101.

(29) Gould, T.; Pittalis, S. Density-Driven Correlations in Many-Electron Ensembles: Theory and Application for Excited States. Phys. Rev. Lett. 2019, 123, 016401.

(30) Gould, T.; Pittalis, S. Density Driven Correlations in Ensemble Density Functional Theory: Insights from Simple Excitations in Atoms. Aus. J. Chem.; preprint on chemrxiv 2020,

(31) Fromager, E. Individual correlations in ensemble density-functional theory: State-driven/density-driven decompositions without additional Kohn-Sham systems. 2020.

(32) Loos, P.-F.; Fromager, E. A weightdependent local correlation densityfunctional approximation for ensembles. 2020 .

(33) Marut, C.; Senjean, B.; Fromager, E.; Loos, P.-F. Weight Dependence of Local Exchange-Correlation Functionals in Ensemble Density-Functional Theory: Double Excitations in Two-Electron Systems. 2020

(34) Levy, M. Universal variational functionals of electron densities, first-order density matrices, and natural spin-orbitals and solution of the v-representability problem. Proc. Natl. Acad. Sci. U.S.A. 1979, 76, 6062-6065.

(35) Harris, J. Adiabatic-connection approach to Kohn-Sham theory. Phys. Rev. A 1984, 29, 1648-1659.

(36) Gould, T.; Dobson, J. F. The flexible nature of exchange, correlation, and Hartree physics: Resolving "delocalization" errors in a "correlation free" density functional. J. Chem. Phys. 2013, 138, 014103.

(37) Seidl, A.; Görling, A.; Vogl, P.; Majewski, J. A.; Levy, M. Generalized KohnSham schemes and the band-gap problem. Phys. Rev. B 1996, 53, 3764-3774.

(38) Sharp, R. T.; Horton, G. K. A Variational Approach to the Unipotential ManyElectron Problem. Phys. Rev. 1953, 90, 317-317.

(39) Talman, J. D.; Shadwick, W. F. Optimized effective atomic central potential. Phys. Rev. A 1976, 14, 36-40.

(40) Chai, J.-D.; Head-Gordon, M. Systematic optimization of long-range corrected hybrid density functionals. J. Chem. Phys. 2008, 128, 084106.

(41) Becke, A.; Savin, A.; Stoll, H. Extension of the local-spin-density exchangecorrelation approximation to multiplet states. Theor. Chim. Acta 1995, 91, 147156.

(42) Perdew, J. P.; Burke, K.; ; Ernzerhof, M. Generalized gradient approximation made simple. Phys. Rev. Lett. 1996, 77, 386568 .

(43) Adamo, C.; Barone, V. Toward reliable density functional methods without adjustable parameters: The PBE0 model. J. Chem. Phys. 1999, 110, 6158-69.

(44) Gould, T.; Toulouse, J. Kohn-Sham potentials in exact density-functional theory at noninteger electron numbers. Phys. Rev. A 2014, 90, 050502.

(45) Loos, P.-F.; Jacquemin, D. Chemically Accurate 0-0 Energies with not-soAccurate Excited State Geometries. J. Chem. Theory Comput. 2019,

(46) Lee, J.; Head-Gordon, M. Two singlereference approaches to singlet biradicaloid problems: Complex, restricted 
orbitals and approximate spin-projection combined with regularized orbitaloptimized Møller-Plesset perturbation theory. J. Chem. Phys. 2019, 150, 244106.

(47) Chien, A. D.; Holmes, A. A.; Otten, M.; Umrigar, C. J.; Sharma, S.; Zimmerman, P. M. Excited States of Methylene, Polyenes, and Ozone from Heat-Bath Configuration Interaction. J. Phys. Chem. A 2018, 122, 2714-2722.

(48) Gould, T.; Stefanufucci, G.; Pittalis, S. Private communication. 2020.

(49) Parrish, R. M.; Burns, L. A.; Smith, D. G.; Simmonett, A. C.; DePrince III, A. E.; Hohenstein, E. G.; Bozkaya, U.; Sokolov, A. Y.; Di Remigio, R.; Richard, R. M. et al. Psi4 1.1: An open-source electronic structure program emphasizing automation, advanced libraries, and interoperability. J. Chem. Theory Comput. 2017, 13, 3185-3197.

(50) Smith, D. G.; Burns, L. A.; Sirianni, D. A.; Nascimento, D. R.; Kumar, A.; James, A. M.; Schriber, J. B.; Zhang, T.; Zhang, B.; Abbott, A. S. et al. Psi4NumPy: An interactive quantum chemistry programming environment for reference implementations and rapid development. J. Chem. Theory Comput. 2018, 14, 3504-3511.

(51) Kendall, R. A.; Dunning Jr, T. H.; Harrison, R. J. Electron affinities of the firstrow atoms revisited. Systematic basis sets and wave functions. J. Chem. Phys 1992, 96, 6796-6806. 


\title{
Supplementary Material for "Approximately Self-Consistent Ensemble Density Functional Theory With All Correlations"
}

\author{
Tim Gould \\ Qld Micro- and Nanotechnology Centre, \\ Griffith University, Nathan, Qld 4111, Australia
}

\begin{abstract}
Supplementary material for "Approximately self-consistent ensemble density functional theory with all correlations". Includes: 1) Full working for the implementation of the $\omega$ B97X density functional approximation for ensembles. 2) Details of the density-driven correlation calculation. 3) Tabulated data for calculations.
\end{abstract}




\section{FULL WORKING FOR $\omega$ B97X}

All tests involve an even number $N$ of electrons, and a mixture of ground/singlet- and triplet-states. Thus, only these terms are considered here.

\section{A. The ground-state terms}

The ground/singlet-state Kohn-Sham wavefunction for $N$ electrons is a Slater-determinant formed on $N / 2$ doubly $(\uparrow / \downarrow)$ occupied orbitals. This gives:

$$
\begin{aligned}
n_{s, \mathrm{gs}}(\boldsymbol{r}) & =2 \sum_{i \leq h} \phi_{i}(\boldsymbol{r})^{2}, \\
P_{2, \kappa}\left(\boldsymbol{r}, \boldsymbol{r}^{\prime}\right) & =n_{s, \mathrm{gs}}(\boldsymbol{r}) n_{s, \mathrm{gs}}\left(\boldsymbol{r}^{\prime}\right)-\frac{1}{2} \gamma_{s, \mathrm{gs}}\left(\boldsymbol{r}, \boldsymbol{r}^{\prime}\right)^{2}, \\
\zeta_{s, \mathrm{gs}}(\boldsymbol{r}) & =0
\end{aligned}
$$

for the density, pair-density and spin-polarisation, respectively, where $h=N / 2$ labels the highest occupied molecular orbital (HOMO). Here,

$$
\gamma_{s, \mathrm{gs}}\left(\boldsymbol{r}, \boldsymbol{r}^{\prime}\right)=2 \sum_{i \leq h} \phi_{i}(\boldsymbol{r}) \phi_{i}\left(\boldsymbol{r}^{\prime}\right)
$$

and $n_{s, \mathrm{gs}}(\boldsymbol{r})=\gamma_{s, \mathrm{gs}}(\boldsymbol{r}, \boldsymbol{r})$ is used to find

$$
\zeta=\sqrt{1-2 P_{2, \mathrm{gs}}(\boldsymbol{r}, \boldsymbol{r}) / n_{s, \mathrm{gs}}(\boldsymbol{r})^{2}}=\sqrt{1-2\left(n_{s, \mathrm{gs}}^{2} / 2\right) / n_{s, \mathrm{gs}}^{2}}=0 .
$$

The Hartree-exchange term,

$$
\Lambda_{\mathrm{Hx}, \mathrm{gs}}=\int \frac{d \boldsymbol{r} d \boldsymbol{r}^{\prime}}{2\left|\boldsymbol{r}-\boldsymbol{r}^{\prime}\right|} P_{2, \mathrm{gs}}\left(\boldsymbol{r}, \boldsymbol{r}^{\prime}\right),
$$

is defined by integrating $P_{2}\left(\boldsymbol{r}, \boldsymbol{r}^{\prime}\right)$ with the Coulomb potential. The pure Hartree term is the usual density functional, $E_{\mathrm{H}}[n]=\frac{1}{2} \int d \boldsymbol{r} d \boldsymbol{r}^{\prime} /\left|\boldsymbol{r}-\boldsymbol{r}^{\prime}\right| n(\boldsymbol{r}) n\left(\boldsymbol{r}^{\prime}\right)$. Then, swapping $1 / r$ for $\operatorname{erfc}(\omega r) / r$ (with $\omega=0.3 \mathrm{bohr}^{-1}$ ) in $\Lambda_{\mathrm{Hx}}$ and $E_{\mathrm{H}}$, gives the short-range exchange term:

$$
\Lambda_{\mathrm{x}, \mathrm{gs}}^{\mathrm{sr}}=-\frac{1}{2} \int \frac{\operatorname{erfc}\left(\omega\left|\boldsymbol{r}-\boldsymbol{r}^{\prime}\right|\right)}{2\left|\boldsymbol{r}-\boldsymbol{r}^{\prime}\right|} d \boldsymbol{r} d \boldsymbol{r}^{\prime} \gamma_{s, \mathrm{gs}}\left(\boldsymbol{r}, \boldsymbol{r}^{\prime}\right)^{2}
$$

\section{B. The triplet-state terms}

There are three triplet-state Kohn-Sham wavefunctions, all yielding the same density and energies. For convenience, pick the one involving $N / 2+1$ electrons in the $\uparrow$ channel, and 
$N / 2-1$ electrons in the $\downarrow$ channel, which is (spatially) given by a trivial product of the respective Slater-determinants. This gives:

$$
\begin{aligned}
n_{s, \mathrm{ts}}(\boldsymbol{r}) & =2 \sum_{i<h} \phi_{i}(\boldsymbol{r})^{2}+\phi_{h}(\boldsymbol{r})^{2}+\phi_{l}(\boldsymbol{r})^{2}, \\
P_{2, \mathrm{ts}}\left(\boldsymbol{r}, \boldsymbol{r}^{\prime}\right) & =n_{s, \mathrm{ts}}(\boldsymbol{r}) n_{s, \mathrm{ts}}\left(\boldsymbol{r}^{\prime}\right)-\sum_{\sigma \in\{\uparrow, \downarrow\}} \gamma^{\sigma}\left(\boldsymbol{r}, \boldsymbol{r}^{\prime}\right)^{2}, \\
\zeta_{s, \mathrm{ts}}(\boldsymbol{r}) & =\frac{\phi_{h}(\boldsymbol{r})^{2}+\phi_{l}(\boldsymbol{r})^{2}}{n_{s, \mathrm{ts}}(\boldsymbol{r})}
\end{aligned}
$$

for the density, pair-density and spin-polarisation. Here $l=h+1$ is the lowest unoccupied molecular orbital (LUMO). This involves,

$$
\gamma^{\uparrow}\left(\boldsymbol{r}, \boldsymbol{r}^{\prime}\right)=\sum_{i \leq l} \phi_{i}(\boldsymbol{r}) \phi_{i}\left(\boldsymbol{r}^{\prime}\right), \quad \quad \gamma^{\downarrow}\left(\boldsymbol{r}, \boldsymbol{r}^{\prime}\right)=\sum_{i<h} \phi_{i}(\boldsymbol{r}) \phi_{i}\left(\boldsymbol{r}^{\prime}\right)
$$

Thus, $n \equiv n_{s, \text { ts }}(\boldsymbol{r})=\sum_{\sigma} \gamma^{\sigma}(\boldsymbol{r}, \boldsymbol{r}) \equiv n^{\uparrow}(\boldsymbol{r})+n^{\downarrow}(\boldsymbol{r})$, giving

$$
\zeta=\frac{\sqrt{n^{2}-2\left(n^{2}-n^{\uparrow 2}-n^{\downarrow 2}\right)}}{n}=\frac{\sqrt{n^{2}-4 n^{\uparrow} n^{\downarrow}}}{n}=\frac{\left|n^{\uparrow}-n^{\downarrow}\right|}{n}=\frac{\phi_{h}^{2}+\phi_{l}^{2}}{n_{s, \mathrm{ts}}} .
$$

The Hartree-exchange term,

$$
\Lambda_{\mathrm{Hx}, \mathrm{ts}}=\int \frac{d \boldsymbol{r} d \boldsymbol{r}^{\prime}}{2\left|\boldsymbol{r}-\boldsymbol{r}^{\prime}\right|} P_{2, \mathrm{ts}}\left(\boldsymbol{r}, \boldsymbol{r}^{\prime}\right)
$$

has the same form as for the ground-state. Again, subtract the short-range Hartree energy expression (again, $\omega=0.3 \mathrm{bohr}^{-1}$ ) $E_{\mathrm{H}}^{\mathrm{sr}}\left[n_{s, \mathrm{ts}}\right]$ to get

$$
\Lambda_{\mathrm{x}, \mathrm{gs}}^{\mathrm{sr}}=-\sum_{\sigma \in\{\uparrow, \downarrow\}} \int \frac{\operatorname{erfc}\left(\omega\left|\boldsymbol{r}-\boldsymbol{r}^{\prime}\right|\right)}{2\left|\boldsymbol{r}-\boldsymbol{r}^{\prime}\right|} d \boldsymbol{r} d \boldsymbol{r}^{\prime} \gamma^{\sigma}\left(\boldsymbol{r}, \boldsymbol{r}^{\prime}\right)^{2}
$$

\section{Putting it together}

Using the results found in the previous subections, gives the full $\omega$ B97X ensemble energy functional as:

$$
\begin{aligned}
\mathcal{E}_{\mathrm{Hxc}}^{\omega \mathrm{B} 97 \mathrm{X}}= & w_{\mathrm{gs}} E_{\mathrm{Hx}}\left[n_{s, \mathrm{gs}}\right]+w_{\mathrm{ts}} E_{\mathrm{Hx}}\left[n_{s, \mathrm{ts}}\right] \\
& -0.8423\left[w_{\mathrm{gs}} \Lambda_{\mathrm{x}, \mathrm{gs}}+w_{\mathrm{ts}} \Lambda_{\mathrm{x}, \mathrm{ts}}\right] \\
& +w_{\mathrm{gs}} E_{\mathrm{xc}}^{\mathrm{DFA}}\left[n_{s, \mathrm{gs}}, 0\right]+w_{\mathrm{ts}} E_{\mathrm{xc}}^{\mathrm{DFA}}\left[n_{s, \mathrm{ts}}, \zeta_{s, \mathrm{ts}}\right] .
\end{aligned}
$$

Here, $0.8423=1-c_{\mathrm{x}}=1-0.1577$ is the short-range exchange damping factor. 
The semi-local term is

$$
E_{\mathrm{xc}}^{\mathrm{DFA}}[n, \zeta]=E_{\mathrm{x}}^{\mathrm{sr}-\mathrm{DFA}}\left[n_{\uparrow}, n_{\downarrow}\right]+E_{\mathrm{c}}^{\mathrm{DFA}}\left[n_{\uparrow}, n_{\downarrow}\right]
$$

and is as given by Chai and Head-Gordon[1], for $n_{\uparrow / \downarrow}=n(1 \pm \zeta) / 2$. Note, $\omega$ B97X is originally defined as, $E_{\mathrm{xc}}^{\omega \mathrm{B} 97 \mathrm{X}}=E_{\mathrm{x}}^{\mathrm{lr}}+c_{\mathrm{x}} E_{\mathrm{x}}^{\mathrm{sr}}+E_{\mathrm{xc}}^{\mathrm{DFA}}$. To obtain $(13)$, use $E_{\mathrm{x}}=E_{\mathrm{x}}^{\mathrm{lr}}+E_{\mathrm{x}}^{\mathrm{sr}}$ to write $E_{\mathrm{xc}}^{\omega \mathrm{B} 97 \mathrm{X}}=E_{\mathrm{x}}+\left(c_{\mathrm{x}}-1\right) E_{\mathrm{x}}^{\mathrm{sr}}+E_{\mathrm{xc}}^{\mathrm{DFA}}$, and then add $E_{\mathrm{H}}$.

The ensemble density is,

$$
n(\boldsymbol{r})=w_{\mathrm{gs}} n_{s, \mathrm{gs}}(\boldsymbol{r})+w_{\mathrm{ts}} n_{s, \mathrm{ts}}(\boldsymbol{r})=\sum_{i} f_{i} \phi_{i}(\boldsymbol{r})^{2},
$$

where $f_{i}=2$ for $i<h, f_{h}=2-w_{\mathrm{ts}}, f_{l}=w_{\mathrm{ts}}$ and $f_{i}=0$ otherwise. The KS kinetic energy term is defined similarly, as

$$
\mathcal{T}_{s}=\sum_{i} f_{i} \int \frac{1}{2}\left|\nabla \phi_{i}(\boldsymbol{r})\right|^{2} d \boldsymbol{r}
$$

The final quantity required for the super simple optimized effective potential (SSOEP) calculations is

$$
\frac{n_{\mathrm{gs}^{\prime}}(\boldsymbol{r})}{n(\boldsymbol{r})}=\frac{\sum_{i \leq h} f_{i} \phi_{i}(\boldsymbol{r})^{2}}{\sum_{i \leq l} f_{i} \phi_{i}(\boldsymbol{r})^{2}}=1-\frac{w_{\mathrm{ts}} \phi_{l}(\boldsymbol{r})^{2}}{n(\boldsymbol{r})},
$$

which is used to calculate $\tilde{v}_{s}(\boldsymbol{r})$ [Eq. (11) of the main text]. Note that this term is close to one in the core region of atoms, where neither the HOMO nor LUMO play much role. It also goes to zero for $\boldsymbol{r} \rightarrow \infty$ due to the slower decay of $\phi_{l}$ compared to $\phi_{h}$.

\section{DENSITY-DRIVEN CORRELATIONS}

In recent work[2], Gould and Pittalis showed that EDFT introduces an additional densitydriven correlation energy term to calculations, that is not present in pure-state calculations. This section summarises this formalism, and details the solution for dealing with densitydriven correlations that is proposed in the main text. Similar arguments may also be found in Section IIIA of Ref. 3, but are restricted to exact exchange theory.

We begin by summarising the formal definition of state-driven and density-driven correlations. For a given externel potential $v$, the true energy $\mathcal{E}^{\mathbf{w}}$ of any ensemble system depends only trivially on the weights, via averaging, i.e.

$$
\mathcal{E}^{\mathbf{w}}[v]=\sum_{\kappa} w_{\kappa} E_{\kappa}[v]
$$


Formally, the state-driven energy of the systems studied in this work are defined as[2],

$$
\mathcal{E}_{\mathrm{c}}^{\mathrm{SD}, \mathrm{w}}[v]=\sum_{\kappa} w_{\kappa} E_{\mathrm{c}, \kappa}\left[n_{\kappa}[v]\right]
$$

where $E_{\mathrm{c}, \kappa}\left[n_{\kappa}\right]=E_{\kappa}-\int n_{\kappa} v d \boldsymbol{r}-T_{s, \kappa}\left[n_{\kappa}\right]-\Lambda_{\mathrm{Hx}, \kappa}\left[n_{\kappa}\right]$ is the usual DFT correlation energy functional for density $n_{\kappa}$, with appropriate spin-symmetry accounted for. Eq. (19) involves only the density $n_{\kappa}=\langle\kappa|\hat{n}| \kappa\rangle$ of the interacting system. $E_{\mathrm{c}, \kappa}$ is thus also only trivially dependent (i.e., expliticly through the terms $w_{\kappa}$ but with no additional implicit dependence on $\mathbf{w}$ ) on the ensemble weights, since $n_{\kappa}$ is obtainable directly from the external potential $v$. Note that, unlike the more general case, both $T_{s, \kappa}\left[n_{\kappa}\right]$ and $\Lambda_{\mathrm{Hx}, \kappa}\left[n_{\kappa}\right]$ can be defined uniquely for either Coulomb systems or low-lying excitations. Both conditions are met in the current work. More generally, one must rely on a bifunctional formalism[2].

The Kohn-Sham kinetic $\mathcal{T}_{s}^{\mathrm{w}}=\sum_{\kappa} w_{\kappa} T_{s, \kappa}^{\mathrm{w}}$ and Hartree-exchange $(\mathrm{Hx}) \mathcal{E}_{\mathrm{Hx}}^{\mathrm{w}}=\sum_{\kappa} w_{\kappa} \Lambda_{\mathrm{Hx}, \kappa}^{\mathrm{w}}$ energy functionals have well-defined meanings in ensembles, but depend non-trivially on the full set of ensemble weights. This is because the Kohn-Sham densities $n_{s, \kappa}^{\mathbf{w}}=\left\langle\kappa_{s}^{\mathbf{w}}|\hat{n}| \kappa_{s}^{\mathbf{w}}\right\rangle$, and the orbitals $\phi_{i}^{\mathbf{w}}$ from which they are formed, carry a non-trivial dependence on $\mathbf{w}$. The density-driven correlation energy

$$
\mathcal{E}_{\mathrm{c}}^{\mathrm{DD}, \mathrm{w}}=\sum_{\kappa} w_{\kappa} E_{\mathrm{c}, \kappa}^{\mathrm{DD}, \mathrm{w}} \equiv \mathcal{F}^{1}-\mathcal{T}_{s}-\mathcal{E}_{\mathrm{Hx}}-\mathcal{E}_{\mathrm{c}}^{\mathrm{SD}},
$$

is then defined as the missing correlation energy. Matching terms gives[2]

$$
E_{\mathrm{c}, \kappa}^{\mathrm{DD}, \mathbf{w}}=T_{s, \kappa}\left[n_{\kappa}\right]-T_{s, \kappa}\left[n_{s, \kappa}^{\mathbf{w}}\right]+\Lambda_{\mathrm{Hx}, \kappa}\left[n_{\kappa}\right]-\Lambda_{\mathrm{Hx}, \kappa}\left[n_{s, \kappa}^{\mathbf{w}}\right] .
$$

as the difference between the Hartree-Fock-like energy taken at the interacting density $n_{\kappa}$, and its equivalent taken at the corresponding Kohn-Sham density $n_{s, \kappa}^{\mathrm{w}}$. It thus inherits a non-trivial dependence on $\mathbf{w}$.

Now, let us move from formalism to practicalities. When a density functional approximation (DFA) is made for $E_{\mathrm{Hxc}, \kappa}$, as done here for $\omega \mathrm{B} 97 \mathrm{X}$, two correlation approximations are introduced in ensembles: (i) the DFA itself; (ii) that $E_{\mathrm{c}, \kappa}^{\mathrm{DFA}}\left[n_{\kappa}\right] \approx E_{\mathrm{c}, \kappa}^{\mathrm{DFA}}\left[n_{s, \kappa}^{\mathbf{w}}\right]$, because $n_{\kappa}$ is unknown. It thus makes sense to redefine:

$$
\begin{aligned}
& E_{\mathrm{Hxc}, \kappa}^{\mathrm{SD}^{\prime}, \mathbf{w}}=T_{s, \kappa}+\Lambda_{\mathrm{Hx}, \kappa}+E_{\mathrm{c}, \kappa}\left[n_{s, \kappa}^{\mathbf{w}}\right], \\
& E_{\mathrm{c}, \kappa}^{\mathrm{DD}, \mathbf{w}}=E_{\mathrm{c}, \kappa}^{\mathrm{DD}, \mathbf{w}}+E_{\mathrm{c}, \kappa}\left[n_{\kappa}\right]-E_{\mathrm{c}, \kappa}\left[n_{s, \kappa}^{\mathbf{w}}\right]
\end{aligned}
$$


using the practical correlation terms, $E_{\mathrm{c}, \kappa}\left[n_{s, \kappa}^{\mathbf{w}}\right]$, where $E_{\mathrm{c}, \kappa}[n]$ is the DFA for correlation, adapted to state $|\kappa\rangle$. Thus, the additional error from (ii) is included in the DD' term, rather than the SD' term. The sum of the two terms remains the same, so nothing additional is lost.

This alternative definition is used in the main paper. In practice, the differences $E_{\mathrm{c}, \kappa}\left[n_{\kappa}\right]-$ $E_{\mathrm{c}, \kappa}\left[n_{s, \kappa}^{\mathbf{w}}\right]$ between SD/SD' and DD/DD' terms (which are equal and opposite, meaning the DD physics is included regardless) are expected to be very small in most systems, due to the semi-local approximation employed in the correlation DFA. Certainly they are likely to be smaller than the error from (i). It thus makes sense to assume that primed and unprimed quantities are essentially equivalent, i.e. that $\mathcal{E}_{\mathrm{Hxc}}^{\mathrm{SD}} \approx \mathcal{E}_{\mathrm{Hxc}}^{\mathrm{SD}}$ and $\mathcal{E}_{\mathrm{c}}^{\mathrm{DD}} \approx \mathcal{E}_{\mathrm{c}}^{\mathrm{DD}^{\prime}}$ and drop primes.

Now, consider the two-state ensemble used in the main text. Its energy is $\mathcal{E}^{w} \equiv$ $\mathcal{E}^{\mathrm{w}=\{1-w, w\}}=(1-w) E_{\mathrm{ss}}+w E_{\mathrm{ts}}$ (i.e. $w_{\mathrm{gs}}=1-w$ and $\left.w_{\mathrm{ts}}=w\right)$. The energy, which is pure-state like for $w=0$ and $w=1$, has no DD correlations for these values. The state-driven energy, $\mathcal{E}^{\mathrm{SD}, w}=\mathcal{F}^{\mathrm{SD}, w}+\int n v d \boldsymbol{r}$, invokes

$$
\begin{aligned}
\mathcal{F}^{\mathrm{SD}, w} & =\mathcal{T}_{s}^{w}+\mathcal{E}_{\mathrm{Hxc}}^{\mathrm{SD}, w} \\
& =w\left[T_{s, \mathrm{gs}}\left[n_{s, \mathrm{gs}}^{\mathbf{w}}\right]+E_{\mathrm{Hxc}, \mathrm{gs}}^{\omega \mathrm{B} 97 \mathrm{X}}\left[n_{s, \mathrm{gs}}^{\mathbf{w}}\right]\right]+(1-w)\left[T_{s, \mathrm{ts}}\left[n_{s, \mathrm{ts}}^{\mathbf{w}}\right]+E_{\mathrm{Hxc}, \mathrm{ts}}^{\omega \mathrm{B} 97 \mathrm{X}}\left[n_{s, \mathrm{ts}}^{\mathbf{w}}\right]\right]
\end{aligned}
$$

Therefore, $\mathcal{E}^{\mathrm{SD}, w}$ is directly calculable using the DFA machinery described in the previous section, using eq. (13). $\mathcal{E}^{w}$ is, in general, unknown but obeys (up to errors from the DFA) $\mathcal{E}^{0}=\mathcal{E}^{\mathrm{SD}, 0}=\mathcal{E}^{\omega \mathrm{B} 97 \mathrm{X}, 0}$ and $\mathcal{E}^{1}=\mathcal{E}^{\mathrm{SD}, 1}=\mathcal{E}^{\omega \mathrm{B} 97 \mathrm{X}, 1}$.

The energy difference $\Delta E=E_{\mathrm{ts}}-E_{\mathrm{ss}}=\mathcal{E}^{1}-\mathcal{E}^{0}$ is the goal of these calculations. $\Delta E$ can be found (formally) by taking derivative $\partial_{w} \mathcal{E}^{w}$ at any $w$, since the energy is linear in $w$. The state-driven energy difference can be similarly defined by taking

$$
\begin{aligned}
\Delta E^{\mathrm{SD}, w=0} & =\partial_{w} \mathcal{E}^{\mathrm{SD}, w=0}=\partial_{w} E^{\omega \mathrm{B} 97 \mathrm{X}, w=0} \\
& \equiv\left[T_{s, \mathrm{ts}}+E_{\mathrm{Hxc}, \mathrm{ts}}^{\omega \mathrm{B} 97 \mathrm{X}}\right]-\left[T_{s, \mathrm{gs}}+E_{\mathrm{Hxc}, \mathrm{gs}}^{\omega \mathrm{B} 97 \mathrm{X}}\right]+\int v\left[n_{s, \mathrm{ts}}-n_{s, \mathrm{ss}}\right] d \boldsymbol{r}
\end{aligned}
$$

at $w=0$, which is similar to the common practice of performing EDFT using the density and orbitals of the pure singlet-state $(w=0)$, where $n_{s, \mathrm{gs}}^{w=0}=n_{\mathrm{gs}}^{w=0}$. In practice, (26) is calculated by a finite difference between $w=0$ and $w=0.1$.

The final step is to use known quantities to obtain $\Delta E . \mathcal{E}^{\mathrm{SD}, w} \equiv \mathcal{E}^{\omega \mathrm{B} 97 \mathrm{X}, w}$ can be found for any $w \leq 0.75$, because of the ensemble minimization constraints and degeneracy $\left(w_{\mathrm{ts}, t} \leq w_{\mathrm{gs}}\right.$ gives $w_{\mathrm{ts}}=3 w_{\mathrm{ts}, t} \leq 0.75$ across the three triplet states). [4] Thus, $\mathcal{E}^{1}=\mathcal{E}^{\omega \mathrm{B} 97 \mathrm{X}, 1}$ cannot always 
be calculated directly. However, fitting a quadratic to $\mathcal{E}^{\omega \mathrm{B} 97 \mathrm{X}, w}$ for values of $w<0.75$ allows for extrapolation to $w \rightarrow 1$ (shortened to $\mathcal{E}^{1} \equiv \mathcal{E}^{\omega \mathrm{B} 97 \mathrm{X}, 1}=\operatorname{ext}_{w \rightarrow 1} \mathcal{E}^{\omega \mathrm{B} 97 \mathrm{X}, w}$ ). We choose a quadratic fit as a linear fit would miss the DD terms entirely, and a cubic or higher fit might lead to overfitting. Then,

$$
\begin{aligned}
\Delta E & =\mathcal{E}^{1}-\mathcal{E}^{0} \equiv \operatorname{ext}_{w \rightarrow 1} \mathcal{E}^{\omega \mathrm{B} 97 \mathrm{X}, w}-\operatorname{ext}_{w \rightarrow 0} \mathcal{E}^{\omega \mathrm{B} 97 \mathrm{X}, w} \\
& \equiv \Delta E^{\mathrm{SD}, w=0}+\Delta \mathcal{E}_{\mathrm{c}}^{\mathrm{DD}} .
\end{aligned}
$$

Thus, the missing DD correlation energy contribution must be

$$
\Delta \mathcal{E}_{\mathrm{c}}^{\mathrm{DD}}=\operatorname{ext}_{w \rightarrow 1} \mathcal{E}^{\omega \mathrm{B} 97 \mathrm{X}, w}-\mathcal{E}^{\omega \mathrm{B} 97 \mathrm{X}, 0}-\left.\partial_{w} \mathcal{E}^{\omega \mathrm{B} 97 \mathrm{X}, w}\right|_{w=0}
$$

to ensure that $\left.\partial_{w} \mathcal{E}^{w}\right|_{w=0}=\left.\partial_{w} \mathcal{E}^{w}\right|_{w=1}$ is independent of $w$. This gives Eqs (9) and (10) of the main paper.

\section{TABULATED DATA}

Tables I and II show the data for the TS12 benchmark set. Table III shows the data for the Ozone molecules.

All data here is reported in $\mathrm{kcal} / \mathrm{mol}$, rather than $\mathrm{eV}$, as this more closely aligns with numerical (rather than methodological) precision. Conversion to eV can be done by dividing numbers by 23.06035.

[1] J.-D. Chai and M. Head-Gordon, J. Chem. Phys. 128, 084106 (2008).

[2] T. Gould and S. Pittalis, Phys. Rev. Lett. 123, 016401 (2019).

[3] T. Gould and S. Pittalis, (2019), 10.26434/chemrxiv.9947249.v1.

[4] In fact calculations can be done for $w=1$ in the specific singlet/triplet case considered here. This is not usually the case, however, so is avoided for the purpose of generality.

[5] J. Lee and M. Head-Gordon, arXiv preprint arXiv:1903.11225 (2019).

[6] A. D. Chien, A. A. Holmes, M. Otten, C. J. Umrigar, S. Sharma, and P. M. Zimmerman, J. Phys. Chem. A 122, 2714 (2018). 
TABLE I. TS12@aug-cc-pVQZ data [in kcal/mol]. Exact results from Ref. 5. Reports mean absolute error (MAE), mean signed error (MSE) and root mean square deviation (RMSD).

\begin{tabular}{|c|c|c|c|c|c|c|c|}
\hline System & Exact & EDFT & Err & sdEDFT & Err & DFT & Err \\
\hline $\mathrm{C}$ & 29.14 & 31.89 & 2.75 & 35.14 & 6.00 & 40.16 & 11.02 \\
\hline $\mathrm{NF}$ & 34.32 & 37.35 & 3.03 & 35.14 & 0.82 & 46.44 & 12.12 \\
\hline $\mathrm{NH}$ & 35.93 & 43.28 & 7.35 & 39.53 & 3.60 & 53.34 & 17.41 \\
\hline $\mathrm{NO}^{-}$ & 17.30 & 17.38 & 0.08 & 9.41 & -7.89 & 28.87 & 11.57 \\
\hline $\mathrm{O}_{2}$ & 22.64 & 31.73 & 9.09 & 30.12 & 7.48 & 38.28 & 15.64 \\
\hline $\mathrm{O}$ & 45.37 & 54.23 & 8.86 & 51.46 & 6.09 & 63.38 & 18.01 \\
\hline $\mathrm{PF}$ & 20.27 & 26.91 & 6.64 & 23.22 & 2.95 & 29.49 & 9.22 \\
\hline $\mathrm{PH}$ & 21.90 & 28.89 & 6.99 & 25.73 & 3.83 & 31.38 & 9.48 \\
\hline $\mathrm{S}_{2}$ & 13.44 & 17.21 & 3.77 & 16.32 & 2.88 & 23.22 & 9.78 \\
\hline S & 26.41 & 33.15 & 6.74 & 32.00 & 5.59 & 38.28 & 11.87 \\
\hline $\mathrm{SO}$ & 18.16 & 22.80 & 4.64 & 21.34 & 3.18 & 28.24 & 10.08 \\
\hline $\mathrm{Si}$ & 18.01 & 26.62 & 8.61 & 21.34 & 3.33 & 25.10 & 7.09 \\
\hline MAE & & & 5.71 & & 4.47 & & 11.94 \\
\hline MSE & & & 5.71 & & 3.15 & & 11.94 \\
\hline RMSD & & & 6.32 & & 4.91 & & 12.37 \\
\hline
\end{tabular}


TABLE II. As Table I but for aug-pVTZ [in kcal/mol].

\begin{tabular}{l|rrrrrrr}
\hline \hline System & Exact & EDFT & Err & sdEDFT & Err & DFT & Err \\
\hline $\mathrm{C}$ & 29.14 & 32.13 & 2.99 & 35.77 & 6.63 & 40.79 & 11.65 \\
$\mathrm{NF}$ & 34.32 & 37.56 & 3.24 & 35.14 & 0.82 & 45.81 & 11.49 \\
$\mathrm{NH}$ & 35.93 & 43.30 & 7.37 & 39.53 & 3.60 & 52.71 & 16.78 \\
$\mathrm{NO}^{-}$ & 17.30 & 19.52 & 2.22 & 15.69 & -1.61 & 29.49 & 12.19 \\
$\mathrm{O}_{2}$ & 22.64 & 31.54 & 8.90 & 30.75 & 8.11 & 37.65 & 15.01 \\
$\mathrm{O}$ & 45.37 & 54.15 & 8.78 & 52.08 & 6.71 & 62.75 & 17.38 \\
$\mathrm{PF}$ & 20.27 & 27.08 & 6.81 & 23.22 & 2.95 & 29.49 & 9.22 \\
$\mathrm{PH}$ & 21.90 & 28.83 & 6.93 & 25.73 & 3.83 & 31.38 & 9.48 \\
$\mathrm{~S}_{2}$ & 13.44 & 17.57 & 4.13 & 16.32 & 2.88 & 23.22 & 9.78 \\
$\mathrm{~S}$ & 26.41 & 33.23 & 6.82 & 32.00 & 5.59 & 38.28 & 11.87 \\
$\mathrm{SO}$ & 18.16 & 23.28 & 5.12 & 21.34 & 3.18 & 28.24 & 10.08 \\
$\mathrm{Si}$ & 18.01 & 26.51 & 8.50 & 21.96 & 3.95 & 24.47 & 6.46 \\
\hline $\mathrm{MAE}$ & & & 5.98 & & 4.15 & & 11.78 \\
$\mathrm{MSE}$ & & & 5.98 & & 3.89 & & 11.78 \\
$\mathrm{RMSD}$ & & & & & 4.65 & & 12.18 \\
\hline \hline
\end{tabular}


TABLE III. Energetics of the open minimum (OM), transition state (TS) and ring minimum (RM) structures of ozone, shown relative to the TM groundstate. All energies in $\mathrm{kcal} / \mathrm{mol}$. Benchmark data from Ref. 6. Calculations used the aug-cc-pVTZ basis set. To maintain consistency, the EDFT gs and ts are both obtained using the same quadratic (with $w=0$ and $w=1$ ). This can lead to differences in the ground-state energy between DFT and EDFT.

\begin{tabular}{|c|c|c|c|c|c|c|}
\hline & $\mathrm{OM}$ & Err & $\mathrm{TS}$ & Err & $\mathrm{RM}$ & Err \\
\hline \multicolumn{7}{|c|}{ Benchmark } \\
\hline gs & 0 & - & 55.6 & - & 30.0 & \\
\hline ts & 95.2 & - & 55.8 & - & 171 & \\
\hline \multicolumn{7}{|l|}{ EDFT } \\
\hline gs & 0 & - & 69.5 & 14.0 & 26.4 & -3.6 \\
\hline ts & 37.6 & -57.6 & 58.6 & 2.8 & 121 & -50.4 \\
\hline ts $(\mathrm{sd})$ & 37.7 & -57.6 & 178 & 122 & 122 & -49.6 \\
\hline \multicolumn{7}{|l|}{ DFT } \\
\hline gs & 0 & - & 64.6 & 9.1 & 26.4 & -3.6 \\
\hline ts & 20.7 & -74.5 & 34.5 & -21.3 & 94.8 & -76.6 \\
\hline
\end{tabular}

